\title{
Component Placement Optimization in the Brain
}

\author{
Christopher Cherniak \\ Committee on History and Philosophy of Science, Department of Philosophy, University of Maryland, College Park, \\ Maryland 20742
}

\begin{abstract}
This computational neuroanatomy study evaluates how well some formalisms derived from combinatorial network optimization theory fit as models for brain structure. At multiple hierarchical levels-brain, ganglion, individual cell-physical placement of neural components appears consistent with a single, simple goal: minimize cost of connections among the components. The most dramatic instance of this "save wire" organizing principle is reported for adjacencies among ganglia in the nematode nervous system; among about $40,000,000$ alternative layout orderings, the actual ganglion placement in fact requires the least total connection length. In addition, evidence supports a component placement optimization hypothesis for positioning of individual neurons in the nematode, and also for positioning of mammalian cortical areas.
\end{abstract}

[Key words: computational neuroanatomy, network optimization, component placement, connectivity, Caenorhabditis elegans, cerebral cortex, adjacency rule]

A basic problem of network optimization theory is, for the connections among a set of components, to determine the spatial layout of the components that minimizes total connection costs. This simple goal seems to account for nervous system anatomy at several organizational levels. It explains "why the brain is in the head" of vertebrates and invertebrates - this placement in fact minimizes total nerve connection lengths to and from the brain. Proceeding to the internal structure of the brain, the working hypothesis of component placement optimization in cerebral cortex is consistent with known interconnections and spatial layout of cat visual and rat olfactory areas. In addition, the hypothesis exactly predicts contiguities among ganglia in the Caenorhabditis elegans nervous system. Finally, this "brain as ultimate VLSI chip" framework also applies to the lowest-level components, to predict grouping of individual neurons of the nematode into ganglion clusters, and even their positioning within ganglia. The observed harmony of component placement and connections in turn raises questions about whether in fact connections lead to optimal positioning of components, or vice versa.

\footnotetext{
Received May 1, 1992; revised Oct. 1, 1993; accepted Oct. 12, 1993.

Software for exhaustive search of C. elegans ganglion layouts was written by Du Won Kang. Preliminary versions of the AY atlas of C. elegans neuroanatomy were generously made available by Theodore Achacoso and William Yamamoto. This research was supported in part by the University of Maryland Institute for Advanced Computer Studies, and by National Institute of Mental Health Grant MH49867.

Correspondence should be addressed to Christopher Cherniak at the above address

Copyright (c) 1994 Society for Neuroscience $0270-6474 / 94 / 142418-10 \$ 05.00 / 0$
}

\section{Component placement optimization}

When a quantitative assessment of functional capabilities of the human brain is compared with an inventory of its neuroanatomical hardware, the conclusion emerges that neurocomputational resources for our cognitive capacities are stringently constrained (Cherniak, 1986, 1988, 1990b, 1991). In particular, on an assumption that in some sense the abstract function of the brain is to make connections, it becomes evident that the physical, neural connectivity to accomplish this is critically scarce. The formalism of scarcity of connections is combinatorial network optimization theory. For instance, if there is strong pressure to optimize deployment of the brain's limited neurowiring, the formal concept of a Steiner tree (Bern and Graham, 1989) should provide a model for the most parsimonious geometry of dendritic and axonic arbors to interconnect a set of synapses with a cell body; and in fact, actual dendrite and axon anatomy at the local level turns out to approximate well Steiner tree junctions (Cherniak, 1990a, 1992). In exploring the hypothesis that the simple generative rule "save wire" drives a significant portion of the physical configuration of the brain, one can go on to ask how well neuroanatomy conforms to other network optimization ideas.

One of these optimization concepts is a kind of mirror-image of the Steiner tree problem - interconnections among a set of nodes are treated as fixed in advance, but the physical locations of those nodes can now be moved around for the best solution. The component placement optimization problem (CPO) is: given the connections among a set of components, find the spatial layoul of the components that minimizes total connection costs (e.g., total length). The problem, as "facility layout," has a long tradition in operations research (e.g., Francis and White, 1974), for instance, in devising the most efficient floor plan for a factory when the flow of materials from one manufacturing operation to another is known. The problem received further attention with the emergence of circuit placement tasks for design of very large scale integrated (VLSI) microcircuit chips (Soukup, 1981; Kuh and Ohtsuki, 1990).

The working hypothesis examined herc is that, bccause neural connectivity resources-particularly long-range connectionsare so severely constrained, component placement optimization is a strong organizing principle for the structure of the nervous system at all hierarchical levels. (1) At the highest level, minimizing total nerve length costs accounts for location of the brain in the bodies of both vertebrates and invertebrates. (2) Component placement to optimize fiber tract lengths explains positioning of functional areas on the cerebral cortex, as well as the physical layout of ganglia in invertebrate nervous systems. (3) At the lowest level, connection length-minimization predicts 
both grouping and positioning of individual neurons in higherorder structures such as ganglia. Costs of longer neural connections include not only the volume of highly metabolically active tissue to be grown and maintained, but also increased signal propagation delays, since neuron conduction velocities are slow. Although cascs of biological optimization to near-absolute physical limits are not unknown (e.g., sensitivity of the dark-adapted human retina and of the human eardrum), the idea may not seem prima facie very plausible that neural connection costs are so severe that simple component placement to minimize those costs would dominate the many other complex demands-on electrophysiological mechanisms, information processing, and so on-involved in design of the best of all possible brains. Nonetheless, checking the CPO hypothesis against a variety of neuroanatomical data is in principle straightforward, with one holistic methodological proviso: that the best tests of the hypothesis will include at least a significant sample of a system at a given level of organization, not just a few isolated elements and interconnections.

\section{Results}

\section{Brain position}

The simplest single-element placement problem is where the human brain is the only locatable component. It is a basic fact of body layout of vertebrates and most actively motile invertebrates that they tend to "lead with their head," to concentrate sensors at this anterior pole; here we treat locations of all sensors and effectors connected to the brain just as prior "edge-constraints" fixed by natural selection. Vertebrate, and most motile invertebrate, bodies have a strongly distinct longitudinal axis. Hence the component placement task here is further simplified, because it approximates a one-axis linear array problem. One issue concerns how connection costs should be assessed. For instance, should the measure be total volume of nerve tract tissue, or total length of individual nerve fibers? Since human nerve fibers range in diameter from below $0.2 \mu \mathrm{m}$ for olfactory fibers to over $10 \mu \mathrm{m}$ for some types of spinal motor fibers (Cherniak, 1990b), the answer significantly affects cost estimates. We begin with the hypothesis that the cost being minimized is individual connection lengths - that is, total fiber length - rather than just volume of tissue. We also start from the assumption that, if connections in themselves count, then sensory and motor connections qualitatively have equal value.

The basic observation concerning component location is that the brain, or predominant portion of most nondiffuse invertebrate nervous systems, is positioned as far forward as is physically possible on the longitudinal axis of the body. Such a placement minimizes total sensory/motor connection costs only if there are more connections to and from the front than the rear. Table 1 gives fiber counts for all cranial nerves and the spinal cord of adult Homo sapiens: the anteroposterior connection ratio for our brain exceeds 5. Despite the flexures of the human CNS, one can therefore see that any placement of the brain other than as far forward as possible will increase total length of required external connections. Some sense of just how strong the selective pressure to "save wire" might be is conveyed when one contemplates how poor a choice in other important respects putting the brain in the head is. Locating the brain instead inside the thorax would greatly improve mechanical protection of this most delicate of tissues, and also save significantly on arteriovenous connection costs to the heart of this most metabolically active of organs.

\begin{tabular}{lr}
\hline Table 1. Anteroposterior connection ratio for the human brain \\
& Fibers (both sides) \\
\hline Cranial nerves & \\
Olfactory & $10,000,000$ \\
Optic & $2,000,000$ \\
Oculomotor & 60,000 \\
Trochlear & 6,000 \\
Trigeminal & 300,000 \\
Abducens & 14,000 \\
Facial & 20,000 \\
Cochlear & 60,000 \\
Vestibular & 40,000 \\
Glossopharyngeal & 7,000 \\
Vagus & 70,000 \\
Accessory & 7,000 \\
Hypoglossal & 15,000 \\
Total & $12,599,000$ \\
Spinal cord & \\
Dorsal & $2,000,000$ \\
Ventral & 400,000 \\
Total & $2,400,000$ \\
\hline
\end{tabular}

Anterior connections exceed posterior ones (ratio anterior: posterior connections $=5.25$ ). Compiled from Blinkov and Glezer (1968) and Shepherd (1979).

The invertebrate nervous system we will be focusing upon is that of the roundworm Caenorhabditis elegans. Two decades of electron microscopy (Albertson and Thomson, 1976; White et al., 1976, 1986; Wood, 1988) have yielded knowledge of the neuroanatomy of this $1.3-\mathrm{mm}$-long free-living soil nematode that is more complete than for any other organism. However, in interpreting connection counts here and below, it should be noted that lacunae (particularly for pharynx and posterior ventral cord), ambiguities, and minor inconsistencies in the published anatomy preclude general error rates below at least a few percent. The nervous system of the hermaphrodite contains 302 neurons, of which the majority, $63 \%$, are located in the most anterior portion of the body, around and just behind the pharynx (see Fig. 1). If this low level of cephalization is loosely termed a brain, we can again ask whether placement of this component is consistent with the CPO hypothesis. The reasoning parallels that for placement of the human brain in the body: Table 2 shows that individual sensory and motor connections to and from the anterior of the worm's body exceed those for the $90 \%$ of the body that is posterior to the concentration of the nervous system in the head by a factor of about 1.5 . Hence, anterior placement of the worm's brain in fact minimizes total costs of interconnections from the sensors and to the muscles of the body.

For no other organism besides $H$. sapiens and $C$. elegans does there presently appear to be adequate quantitative anatomical data to calculate the anteroposterior connection ratio. However, for many species, qualitative estimation of simply whether anterior connections exceed posterior ones is feasible. Examination of a representative vertebrate series does suggest that each animal has an anteroposterior brain connection ratio exceeding 1 -and forward placement of the brain-in conformance with a fiber-minimization hypothesis. Similarly, inspection of more detailed drawings of the much greater variety of invertebrate nervous systems suggests no counterexamples where anterior 


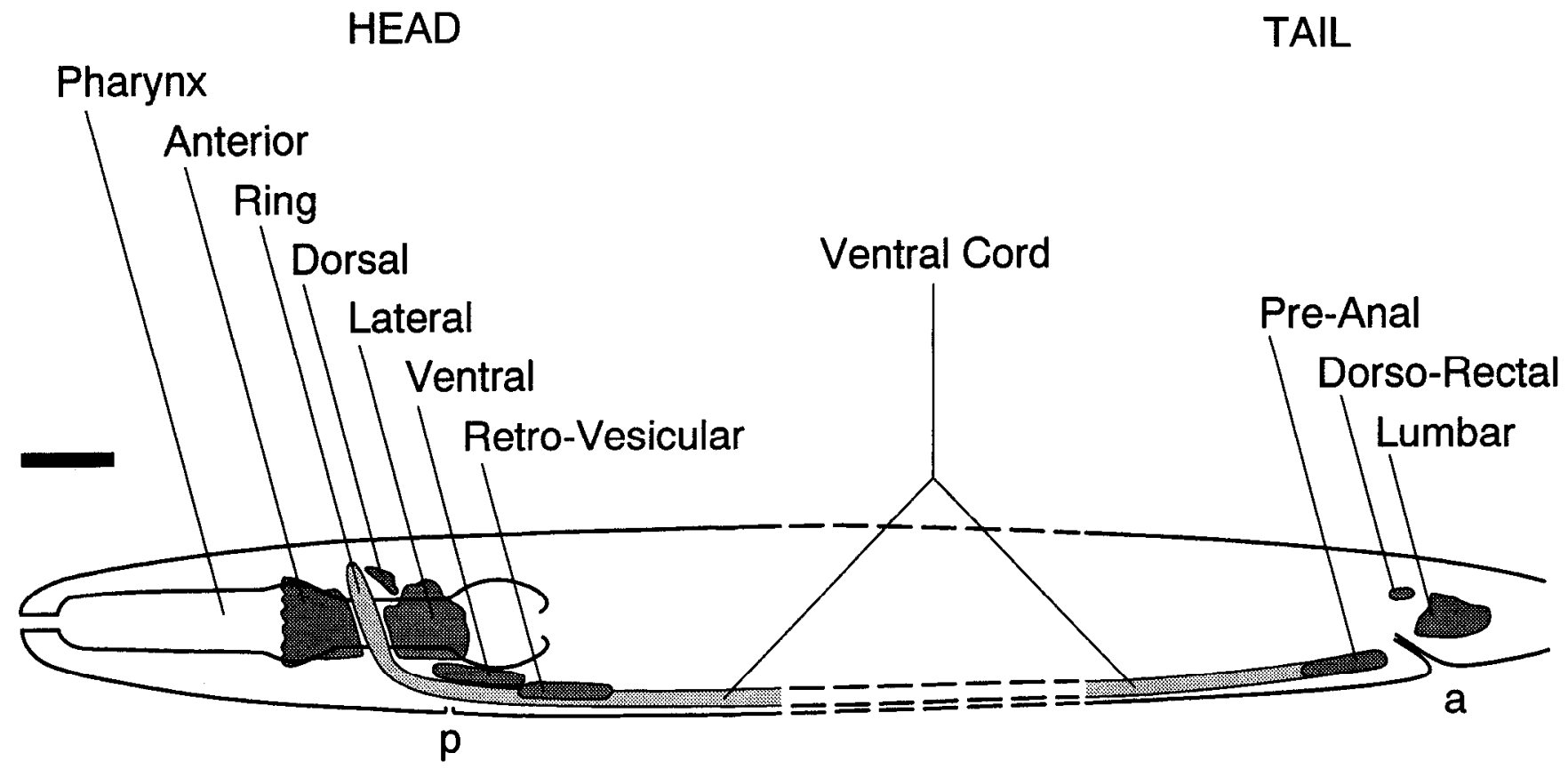

Figure 1. Diagram showing ganglia of Caenorhabditis elegans hermaphrodite: their body locations and schematized shapes. The ganglia are positioned approximately end-to-end, with partial overlap of some contiguous ganglia. The neuron group associated with the pharynx and the ventral cord neuron group are treated here as ganglia. The diffuse pharynx "ganglion" envelops the pharynx musculature and follows its contours; it is not shown separately from the pharynx muscle tube. The anterior and lateral ganglia and the ring in turn surround portions of the pharynx muscles and neurons. The small dorsal ganglion lies partially above the lateral ganglion, and some of the lateral ganglion lies above the anterior portion of the ventral ganglion. The anterior, dorsal, and lateral ganglia directly abut the ring neuropil. The body cavity dorsal to the ventral cord is occupied by the viscera. In the tail, some of the small dorsorectal ganglion lies over the anterior portion of the lumbar ganglion. Not shown: nonganglionic neurons, sensors, nerve process tracts besides the ventral cord. For neuron counts of ganglia, see Table 4 . Total length of the body is $\sim 1300 \mu \mathrm{m}$. Fiducial points: $p$, excretory pore; $a$, anus. Based in part on White et al. $(1976,1986)$, Albertson and Thompson (1976), and Wood (1988). Scale bar, $50 \mu \mathrm{m}$.

\section{Table 2. Anteroposterior connection ratio for "brain" of $C$. elegans}

Fibers

\section{Anterior connections}

Labial processes (6)

Amphid sensilla, etc.

Deirid sensilla, etc.

Pharynx ganglion connections

Head/neck motor connections

Total

44
34
4
24
38
146

Posterior connections

Dorsal ganglion

Lateral ganglion $\quad 16$

Ventral ganglion $\quad 15$

Retrovesicular ganglion $\quad 12$

Ventral cord 14

Preanal ganglion 2

Dorsorectal ganglion 3

Lumbar ganglion $\quad 23$

Nonganglionic

Total

96

"Brain": pharynx, anterior, dorsal, lateral, ventral, retrovesicular ganglia (+ nonganglionic head neurons) $=189$ neurons. Anterior connections exceed posterior ones (ratio anterior: posterior connections $=1.52$ ). Compiled from White et al. (1986) and Wood (1988). connections exceeding posterior ones to the preponderance of the nervous system is not accompanied by forward placement of it.

\section{Cerebral cortex layout}

Passing over the organizational level of the vertebrate brain in terms of cerebrum, midbrain, cerebellum, medulla, we now analyze placement of subdivisions of the cerebral cortex. Topologically, the human cortex is a highly folded shect of around $160,000 \mathrm{~mm}^{2}$, which has been parcellated into over 50 cytoarchitectonically and functionally distinct areas under schemes of the Brodmann and/or von Bonin and Bailey type. During the last decade, extensive compendia have appeared enumerating connections among these areas as revealed by degeneration and pro- and retrograde tracer techniques, mostly focused on connections from and to primary sensory areas of a given modality, such as vision. None of these connectivity compilations purports to be complete; an unknown extent of terra incognita remains. However, one can still check the consistency of a CPO hypothesis for layout of cortical areas-a "plate tectonics of the cortex" - with available partial information on cortical connectivity.

Another issue concerns choice of a usable measure of cost of a connection between a pair of cortical areas. The most realistic estimate would be based on number of fibers and actual length, or shortest possible length, for a fiber tract interconnecting two areas (including necessary detours beneath sulci, etc.). Although Krieg (1963) begins to convey a picture of disposition of some 
Table 3. Connections and contiguities between neural components

\begin{tabular}{|c|c|c|c|c|c|c|c|c|c|}
\hline & \multirow{2}{*}{\multicolumn{3}{|c|}{$\begin{array}{l}\text { Cat visual cortex } \\
\text { areas (18) } \\
\text { Contiguous pairs }\end{array}$}} & \multirow{2}{*}{\multicolumn{3}{|c|}{$\begin{array}{l}\text { Rat olfactory } \\
\text { cortex areas (21) } \\
\text { Contiguous pairs }\end{array}$}} & \multirow{2}{*}{\multicolumn{3}{|c|}{$\begin{array}{l}\text { C. elegans } \\
\text { ganglia (11) } \\
\text { Contiguous pairs }\end{array}$}} \\
\hline & & & & & & & & & \\
\hline & Yes & No & Total & Yes & No & Total & Yes & No & Total \\
\hline \multicolumn{10}{|c|}{ Connected pairs } \\
\hline Yes & 70 & 108 & 178 & 19 & 10 & 29 & 31 & 40 & 71 \\
\hline No & 0 & 128 & 128 & 61 & 330 & 391 & 9 & 59 & 68 \\
\hline Total & 70 & 236 & 306 & 80 & 340 & 420 & 40 & 99 & 139 \\
\hline \multicolumn{10}{|l|}{ Significance } \\
\hline of effect & \multicolumn{3}{|c|}{$p<0.0001$} & \multicolumn{3}{|c|}{$p<0.0001$} & \multicolumn{3}{|c|}{$p<0.0001$} \\
\hline \multicolumn{10}{|l|}{ Magnitude } \\
\hline of effect & \multicolumn{3}{|c|}{$r_{\phi}=0.46$} & \multicolumn{3}{|c|}{$r_{\phi}=0.32$} & \multicolumn{3}{|c|}{$r_{\phi}=0.34$} \\
\hline
\end{tabular}

There is a tendency to conform to the adjacency rule: For each system, a significant proportion more of connected than nonconnected pairs are contiguous. Cat cortex data are derived from Rosenquist (1985); rat cortex data from Price (1987); $C$. elegans data from White et al. $(1976,1986)$, Albertson and Thompson (1976), and Wood (1988). Where $N$ is sample size, magnitude of experimental effect $r_{\phi}=\sqrt{\chi^{2 / N}}$. See Results for connection-counting conventions.

of the human cerebral white-matter tracts, such information in general is not available at the current state of knowledge, and must await development of large-scale neuroanatomy databases. A next-best approximation would be the shortest-distance direct line in three-dimensional space between two areas on the intact cerebrum surface. However, the simplest measure to explore is just a test of the two-dimensional topology of the cortical sheet, such as whether or not two areas are in fact contiguous. Unfolded cortical maps for macaque and cat (Van Essen and Maunsell, 1980), and for human cortex (Jouandet et al., 1989), make assessment of such adjacencies straightforward. Cortical CPO then becomes a problem of optimal tesselation or tiling of a surface. This may not be an entirely farfetched approximation, in light of the corticogenesis hypothesis of Rakic (1988), where parcellation of the neocortical plate in fact stems from a two-dimensional " $x$ - and $y$-coordinate" proto-map in the prolifcrative surface layer of the embryonic ventricle.

A further difficulty is that, as discussed below, exact verification of an optimal layout for even a few dozen elements is not computationally feasible (particularly a jigsaw puzzle of irregularly shaped elements). However, simple statistical tests of placement optimality can be devised: if we hypothesize that contiguities among cortical areas generally are scarcer than interconnections between areas, then the optimization question emerges: how well is the limited supply of available contiguity allotted? The null hypothesis here is that contiguity is uniformly or randomly distributed between pairs of connected and pairs of unconnected areas. On the other hand, if layout of the cortical mosaic is in fact optimized with respect to connectivity costs, then there should be statistically significant support for an adjacency rule of the form, "If areas $a$ and $b$ are connected, then $a$ and $b$ are contiguous," other things being equal. (This outcome should hold irrespective of whether interconnections among areas in fact drive positioning of areas, or vice versa.)

The visual system of the cat (Rosenquist, 1985) can be analyzed in this way (see Table 3 ). Rosenquist (1985, p 104) shows 18 areas of cat cerebral cortex as having corticocortical visual projections, with 178 connections between these areas. (These are reciprocal projections, not listing self-connections of areas, including connections of unclassified density, excluding extracortical inputs and outputs and interhemispheric connections.) The 18 visual cortical areas can form 306 possible pairings, so actual interconnections occupy $58 \%$ of the total available connectivity matrix. From Rosenquist's cortical maps (1985, p 84), an inventory of contiguities among these visual areas is next compiled; there are 70 total, so connectivities between areas exceed contiguities by about $2.5: 1$. In agreement with the adjacency rule described above, proportionately many more of the connected than nonconnected pairs turn out to be contiguous, with a Fisher exact test of this difference showing it is highly significant, $p<0.0001$.

As corroboration of the adjacency rule finding for cat visual areas, connectivities of the quite different cortical olfactory system of the rat (Price, 1987) can be similarly analyzed (see Table 3 ). In this case contiguities between areas exceed reported connectivities. Nonetheless, despite ambiguities concerning some area boundaries, the actual layout still departs from random placement with respect to the adjacency rulc: the pattern of contiguity of connected olfactory areas is again highly significant, $p<0.0001$. A corresponding analysis of macaque visual cortical areas from the data of Van Essen (1985) can be found in Cherniak (1991) [in addition, a somewhat similar treatment (Young, 1992) of more recent published macaque data of Van Essen has appeared while the present article was under review at this journal]; once more, the adjacency rule is strongly confirmed. Nonetheless, support for the adjacency rule still might result from extraneous factors - for example, a range bias in tending not to detect, or report, longer-distance connections between nonadjacent areas. In addition, it should be emphasized that satisfying the adjacency rule is not sufficient in itself to guarantee optimality; for instance, the rule does not specify how best to allocate contiguity when there are too many interconnections for all connected pairs to be contiguous. A strategy for independent validation of the adjacency rule is to examine placement of all ganglia in an invertebrate nervous system, $C$. elegans, where anatomical knowledge is fairly close to complete.

\section{C. elegans ganglia layout}

The view of the worm neuroanatomists has been that these ganglia or spatial clusterings of cell bodies may simply be brought about by extraneous mechanical factors and without functional significance (White et al., 1986; Chalfie and White, 1988). Is placing of these components in fact consistent with a simple rule, "minimize total interconnecting fiber length"? The nem- 
Table 4. Connectivity matrix for 10 ganglia of $C$. elegans

\begin{tabular}{|c|c|c|c|c|c|c|c|c|c|c|c|c|c|c|}
\hline \multirow[b]{2}{*}{ From } & \multirow{2}{*}{$\begin{array}{l}\text { To: } \\
\text { Head } \\
\text { sens. }\end{array}$} & \multicolumn{7}{|c|}{ Head (187n) } & \multirow[b]{2}{*}{$\begin{array}{l}\mathrm{v} \\
\mathrm{vC} \\
58 \\
\end{array}$} & \multicolumn{3}{|c|}{ Tail (39 n) } & \multirow[b]{2}{*}{$\begin{array}{l}\text { Tail } \\
\text { sens. }\end{array}$} & \multirow[b]{2}{*}{ Totals } \\
\hline & & $\begin{array}{l}\text { PH } \\
20 \\
\end{array}$ & $\begin{array}{l}\text { AN } \\
36\end{array}$ & $\begin{array}{l}\text { RNG } \\
0 \\
\end{array}$ & $\begin{array}{l}\text { DO } \\
6 \\
\end{array}$ & $\begin{array}{l}\mathbf{L A} \\
64 \\
\end{array}$ & $\begin{array}{l}\text { VN } \\
32 \\
\end{array}$ & $\begin{array}{l}\mathbf{R V} \\
20 \\
\end{array}$ & & $\begin{array}{l}\text { PA }^{a} \\
12 \\
\end{array}$ & $\begin{array}{l}\text { DR } \\
3 \\
\end{array}$ & $\begin{array}{l}\text { LU } \\
24\end{array}$ & & \\
\hline Pharynx & & m: 11 & & & & & & & & & & & & \\
\hline PH & (13) & 18.5 & & & & & & & & & & & & 31.5 \\
\hline Anterior & & & & $\mathrm{m}: 12$ & & & & & & & & & & \\
\hline $\mathbf{A N}$ & 32 & 2 & & 36 & & & & & & & & & & 70 \\
\hline Dorsal & & & & & & & & & & & & & & \\
\hline DO & 4 & & & 6 & & & & & $\mathrm{~m}: 1$ & 1 & & & 1 & 12 \\
\hline Lateral & & & & $\mathrm{m}: 10$ & & ps: 4 & & & & & & & & \\
\hline $\mathbf{L A}$ & 28 & & & 64 & & 12 & 2 & 12 & 8 & 5 & 4 & 10.5 & & 145.5 \\
\hline Ventral & & & & $\mathrm{m}: 12$ & & & & & cp: 15 & & & & & \\
\hline $\mathbf{V N}$ & 2 & & & 32 & 3 & 2.5 & & 1.5 & & 2 & 2 & 3 & & 48 \\
\hline Retrovesicular & & & & & & & & $\mathrm{m}: 12$ & & & & & & \\
\hline RV & & & & 8 & & 12 & 5 & 16 & 12 & 2 & 1 & 2.5 & 1 & 59.5 \\
\hline Ventral C & & & & & & & & & $\mathrm{m}: 58$ & & & & & \\
\hline vC & & & & & & & 2 & 13 & 53.5 & 11 & 2 & 2 & & 83.5 \\
\hline Preanal & & & & & & & & & $\mathrm{m}: 1$ & $\mathrm{~m}: 9$ & & & & \\
\hline PA ᄂ & & & & 2 & & 2 & 2 & & 8.5 & 11 & 2 & 4.5 & & 32 \\
\hline Dorsorectal & & & & & & & & & & & $\mathrm{m}: 1$ & & & \\
\hline DR & & & & 3 & & 1 & 3 & 2 & 1 & 3 & 1 & 1 & & 15 \\
\hline Lumbar & & & & & & & & & $\mathrm{m}: 2$ & & & & & \\
\hline $\mathbf{L U}$ & & & & 11 & & 17 & 6 & 10 & 5 & 12 & 10 & 19.5 & 14 & 104.5 \\
\hline Totals & 79 & 20.5 & & 162 & 3 & 46.5 & 20 & 54.5 & 88 & 47 & 22 & 43 & 16 & 601.5 \\
\hline
\end{tabular}

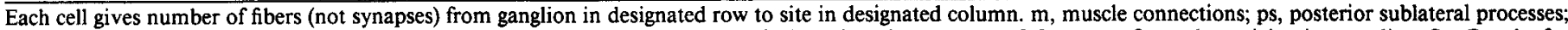

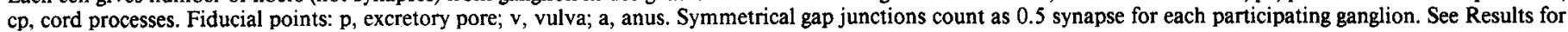
connection-counting conventions.

atode worm nervous system contains 10 ganglia, if the concentrations of cell bodies controlling the pharynx and in the ventral nerve cord are each included; $91 \%$ of the total population of neuron somata are in these ganglia. Except for much of the pharynx grouping, these ganglia are arranged approximately endto-end, with no gaps between them (indeed, with some overlap), from the front of the head to the tail (see Fig. 1). The most distinctive feature of the nervous system is the ring of neuropil cncircling the pharynx, a kind of crossbar network where a large portion of all connections are made-about a third, under the accounting scheme below. The ring must also be classified as a locatable component with the ganglia, making this an 11 element placement problem. The head, neck, and body muscle groups, together with head and tail concentrations of sensors, constitute the main fixed edge-constraints of the problem. The length: diameter aspect ratio of the worm body is about 20:1; hence, except for surrounding muscle groups, to a first approximation of over an order of magnitude, the ganglion layout problem is "roughly one-dimensional."

From the published anatomy (Albertson and Thomson, 1976; White et al., 1976, 1986; Wood, 1988) a connectivity database was constructed: for each individual neuron, all known connections between ganglia, from sensors, and to muscles were listed. This database was checked and supplemented by an early version of the $C$. elegans neuroanatomy database of Achacoso and Yamamoto (1990), independently compiled from similar sources. A connection-counting scheme was devised with the objective of tallying on a consistent and nonarbitrary basis separate fibers running between ganglia.
Rules for counting connections: (1) Two or more chemical synapses from a given neuron to one neuron count as a single (complex) connection, and similarly for multiple neuromuscular junctions. (2) Chemical synapses from a given neuron to more than one neuron in a single ganglion also count as just 1 interor intraganglionic connection. (3) If a neuron makes gap (electrical) synapses to more than one neuron in a ganglion (i.e., there is gap-synapse fan-out), each of these synapses is designated as asymmetrical, like a chemical synapse, with a 1 connection total cost charged to the former, fanning-out neuron as above. (4) Otherwise, a gap-synaptic connection is symmetrical, counting as 0.5 connection for the ganglion of each of the two participating neurons (i.e., the 1 fiber cost of the single gap junction is split between the two ganglia involved). (5) Two or more gap synapses from a given neuron to one neuron count as a single, complex synaptic structure. (6) One chemical plus one or more gap synapses between two neurons count as a single connection to the target ganglion for the chemical synapse. (7) Each two symmetrical gap synapses from a ganglion to different neurons in a ganglion are assigned a summed cost of 1 interconnecting fiber between the ganglia (because each independent gap junction counts by itself as 0.5 fiber).

From the augmented ganglion-level connectivity database, the connectivity matrix of Table 4 was then computed (nonganglionic neurons are not shown). Such a connectivity matrix provides a compact, synoptic representation of the highly complex connectivity relations in even this simple nervous system. Table 4 is semidiagrammatic, with approximate actual anter- 
oposterior order of the ganglia presented. (Qualifications: the lateral ganglion as well as the dorsal ganglion is directly adjacent to the ring; much of the lateral ganglion similarly overlies some of the ventral ganglion; posterior portions of the pharynx group are surrounded by the ring and anterior, dorsal, lateral, and ventral ganglia; and the dorsorectal ganglion lies partly above the lumbar ganglion.) Scale of horizontal extent of ganglia has not been preserved: in common with the ground plan of other roundworms such as Ascaris, ganglia of $C$. elegans form two clusters - a large ("brain") group in the head discussed earlier, and a smaller group in the tail. In between, about $80 \%$ of total body length is occupied by the ventral cord, which is treated here as an elongated ganglionic cluster. Approximate muscle connection locations relative to ganglia have also been indicated, along with sensory processes. A connection between ganglia that proceeds via the ring appears as a connection of each participating ganglion to the ring. The row total for a ganglion can be regarded as the number of fibers leaving the ganglion (not including muscle connections); its column total is then the number of fibers entcring the ganglion (not including mid-body sensory processes).

Inspection of the matrix establishes that even with connectivity information of unprecedented completeness-down to single-fiber interconnections between several pairs of gangliait is not the case that this nervous system constitutes a maximally interconnected network, where "everything is connected to everything"; about half the matrix cells remain empty. The density of interconnection, that is, number of fibers between two ganglia, is as widely and uniformly distributed (from 1 to 64 ) as the neuron population of ganglia (from 3 to 64). An immediately evident trend is distribution of connections along a diagonal axis from the upper left corner to the lower right. This concentration indicates that connections from a given ganglion in fact tend to go to nearby targets. In addition, the matrix can be analyzed as the mammalian cortex data was for agreement with the adjacency rule (see Table 3 ): again, the trend toward adjacency of connections is highly statistically significant (the analysis includes connections to head and tail sensors and to muscles).

The connectivity map of Figure 2 , shown with some compression of its vertical axis, adds further realism by representing the connections of each individual neuron, ganglionic and nonganglionic, as a separate entry. (Ganglion position on the longitudinal axis remains only approximately correct, with the previously discussed qualifications concerning some ganglia overlap.) This compilation appears to provide the first complete depiction of a nervous system at the individual neuron level in a single, synoptic image. Including sensors, muscles, and cell bodies themselves, a total of around 1000 connections appear. It is now possible to identify all instances of fibers skipping connections to intermediate ganglia, that is, where a connection from a given ganglion to a nearby ganglion is not made via a fiber that goes on to connect to a more remote ganglion, but instead via a different fiber that terminates at the nearby ganglion.

Because roundworm ganglia approximate an end-to-end placement scheme (indeed, with some vertical stacking of smallest ganglia), alternative layouts of the ganglia can be represented simply as altcrnativc orderings. For the 11 locatable components in the worm ganglion placement problem, there are 11 ! = $39,916,800$ alternative possible anteroposterior orderings. The actual ganglion order in fact appears to be the unique optimal one out of these millions for minimizing total interconnecting fiber length. This search space can be exhaustively checked: each ganglion layout possibility - with interconnections as in Figure 2 -is generated and evaluated for the total anteroposterior length of its extraganglionic connections (taking into account the anteroposterior spans of the different ganglia, and positions in the worm's body where particular ganglia can overlap-for example, the dorsal and lateral ganglia). For calculation of connection costs, each fiber originating and terminating in a given ganglion is treated as connecting to the "center of mass" of the ganglion. Like sensors and muscles, the nonganglionic neurons remain fixed in their actual locations for this search; there are in fact three times more connections to and from sensors and muscles than to and from the nonganglionic neurons. A sorted list of the best layouts and, concurrently, of the worst ones, is thus produced. When distributed among eleven $20 \mathrm{MHz} 80386$-chip microcomputers and executed in parallel, this brute-force search takes about $50 \mathrm{hr}$.

The worm's actual layout turns out to be the unique best one, requiring $87,803 \mu \mathrm{m}$ of anteroposterior, extraganglionic fiber to make the connections shown in Figure 2. The top six runnerup layouts show no differences from the actual arrangement for ganglia in the head group. The second-best layout uses only 60 $\mu \mathrm{m}$ more connecting fiber than the actual layout, a $<0.1 \%$ difference that suggests quite fine-grained optimization. Connectivity cost of the worst of the top 75 layouts is $3.1 \%$ greater than the actual placement. In fact, a number of the runners-up actually may not be feasibly packable (with local muscles, viscera, etc.) into the worm body; thus, not even any of these impossible layouts turns out to be better than the actual one. An indication of how much is at stake in the worm layout optimizing problem is that the last-place, worst ("pessimal") layout would require almost four times as much connection-length as the optimal one, although again there are questions of its physical packability. (If costs of connections to and from the nonganglionic neurons are excluded from the evaluation, the actual ganglion layout still only drops to 308 th placc among the millions of alternative layouts.)

In interpreting results of this exhaustive search, the sum of connection lengths in a layout can only be viewed as a comparative index of cost; this measure does not include dorsoventral or lateral connection lengths of this slim-bodied creature, or intraganglionic connection costs, which stay fixed for alternative layouts. In addition, plausible error levels in the anatomical database for the search entail care in concluding that the worm ganglion layout is microoptimized. Various simplifying approximations can be further refined - for example, for the sites of origination and termination of connections within a ganglion. Therefore, so far as one can tell, to current limits of accuracy of the anatomical data representation, the actual placement appears to be the best of all possible layouts; this constitutes strong evidence of perfect optimization. (The conclusion is that the actual layout is the one that minimizes total length of fibers; more sensitive cost indices can be compiled-for example, to reflect fiber volume, or weighting of a connection for its number of target neurons.) A conclusion that the actual worm layout, even with some violations of the adjacency rule discussed above, is the fiber-minimizing one brings out the point that perfect, uncompromising solutions to a placement problem often are just not possiblc; for moderately high connectivity densities, there may be no "no-losers" arrangement where every pair of interconnected elements can be contiguous. Such an idea also applies to interpreting the adjacency rule violations for the earlier vertebrate two-dimensional cortical layout problems. 


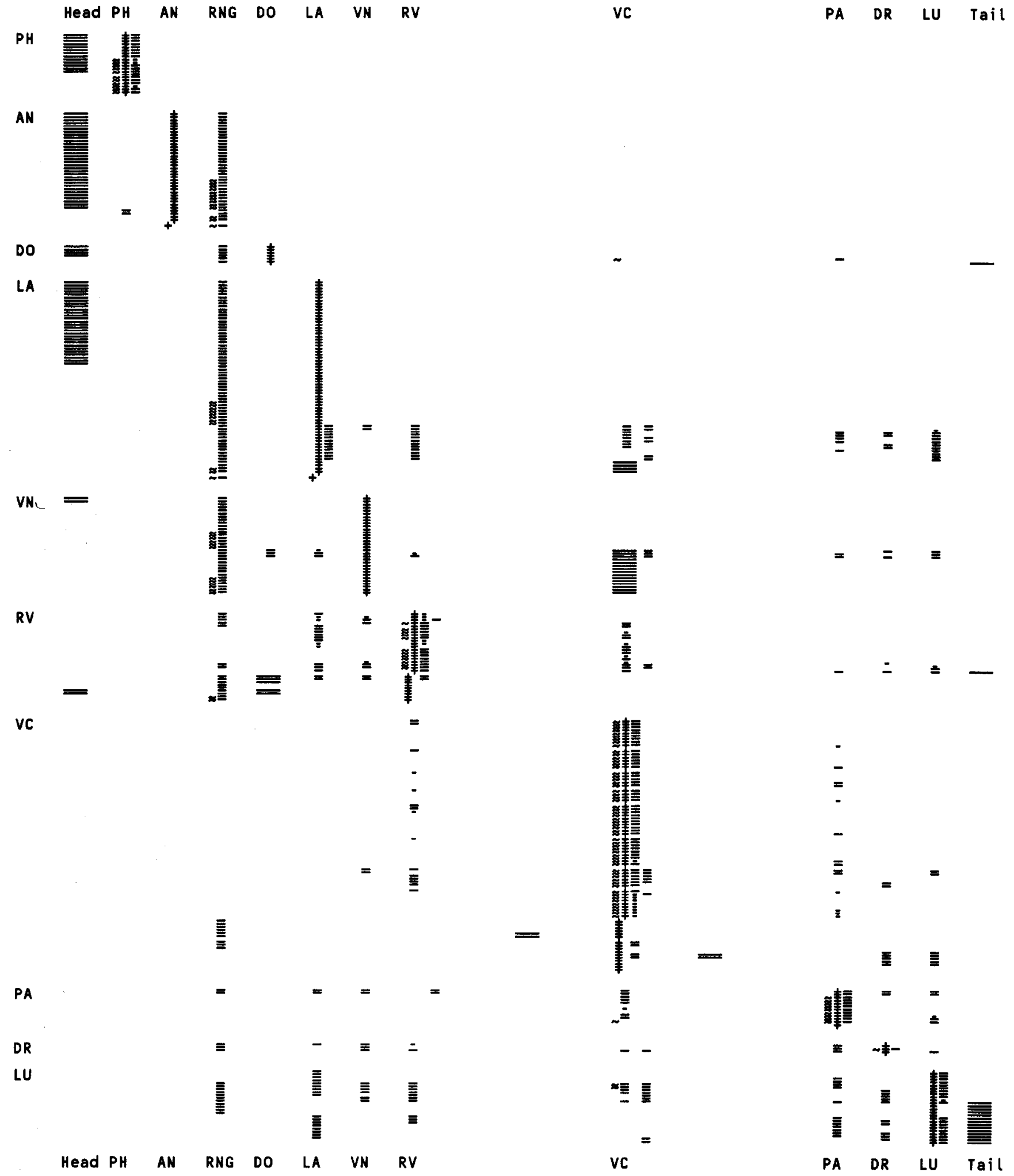

Figure.2. Complete ganglion-level connectivity map for Caenorhabditis elegans nervous system. Each partially superimposed microline represents one of the 302 neurons: + , soma; - , asymmetrical (chemical) synapse; -, symmetrical (gap) synapse; $\sim$, muscle connection; - , sensor. (Nonganglionic somata appear below and one space to left of somata of nearest ganglion; connections to nonganglionic neurons appear in the column one space to right of column for connections to neurons of the nearest ganglion.) Ganglion codes and connection-counting conventions are as in Table 4. An immediately evident trend of distribution of connections around the diagonal from upper left corner to lower right corner indicates that the layout conforms to an adjacency rule that tends to minimize total connection costs. Horizontal scaling, approximately $100 \times$. A hand magnifier and a transparent straight edge will reveal further detail. 
Table 5. Comparison of interconnections versus groupings of neurons by ganglia in C. elegans

\begin{tabular}{|c|c|c|c|c|c|c|c|c|c|c|c|}
\hline \multirow[b]{2}{*}{$\begin{array}{l}\text { Ganglion: } \\
\text { Neurons: }\end{array}$} & \multicolumn{6}{|l|}{ Head } & \multirow[b]{2}{*}{$\begin{array}{l}\mathrm{v} \\
\mathrm{VC} \\
58\end{array}$} & \multicolumn{3}{|l|}{ Tail } & \multirow[b]{2}{*}{$\begin{array}{l}\text { Total } \\
302\end{array}$} \\
\hline & $\begin{array}{l}\text { PH } \\
20\end{array}$ & $\begin{array}{l}\mathbf{A N} \\
36\end{array}$ & $\begin{array}{l}\text { DO } \\
6 \\
\end{array}$ & $\begin{array}{l}\text { LA } \\
64\end{array}$ & $\begin{array}{l}\mathrm{p} \\
\mathbf{V N} \\
32\end{array}$ & $\begin{array}{l}\text { RV } \\
20\end{array}$ & & $\begin{array}{l}\mathbf{P A} \\
12 \\
\end{array}$ & $\begin{array}{l}\text { DR } \\
3\end{array}$ & $\begin{array}{l}\text { LU } \\
24\end{array}$ & \\
\hline \multicolumn{12}{|l|}{ Intraganglionic connections } \\
\hline asym: & $\sim 43$ & 95 & 0 & 300 & 22 & 36 & 159 & 19 & 2 & 49 & \\
\hline sym: & $\sim 10$ & 18 & 2 & 75 & 20 & 17 & 60 & 6 & 0 & 18 & \\
\hline total: & 53 & 113 & 2 & 375 & 42 & 53 & 219 & 25 & 2 & 67 & 951 \\
\hline \multicolumn{12}{|l|}{ Extraganglionic connections } \\
\hline asym: & 0 & 172 & 59 & 446 & 164 & 102 & 42 & 45 & 59 & 204 & \\
\hline sym: & 0 & 24 & 5.5 & 56.5 & 37.5 & 21 & 10 & 9.5 & 8 & 13.5 & \\
\hline total: & 0 & 196 & 64.5 & 502.5 & 201.5 & 123 & 52 & 54.5 & 67 & 217.5 & 1478.5 \\
\hline $\begin{array}{l}\text { Intraganglionic/total } \\
\text { connections }\end{array}$ & 1.000 & 0.366 & 0.030 & 0.427 & 0.172 & 0.301 & 0.808 & 0.314 & 0.029 & 0.236 & $\begin{array}{l}\text { Mean } \\
0.368\end{array}$ \\
\hline $\begin{array}{l}\text { Ganglion/total } \\
\text { neurons }\end{array}$ & 0.066 & 0.119 & 0.020 & 0.212 & 0.106 & 0.066 & 0.192 & 0.040 & 0.010 & 0.079 & $\begin{array}{l}\text { Mean } \\
0.091\end{array}$ \\
\hline
\end{tabular}

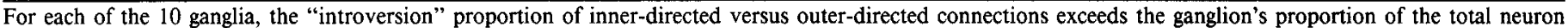

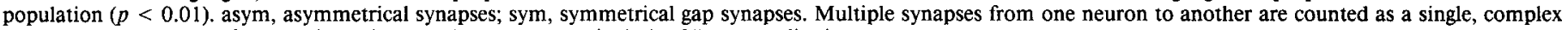
connection. See Results for counting rules. Total neuron count includes 27 nonganglionic neurons.

\section{Individual neuron placement}

In terms of a metaprinciple of similarity of biological structuregenerating rules at different organizational levels, we next explore CPO hypotheses for individual neurons along the lines of what we have found for the total brain and for its ganglion components. That is, treating individual nerve cell components as we did ganglia, we seek evidence of placement of these elements that minimizes connection costs. Again, the most complete information currently available on anatomical positioning of individual neurons is for $C$. elegans. However, a full study of alternative cell layouts like that sketched above for ganglia is not presently feasible (e.g., exhaustive search of all relevant placements of 302 elements would require resources far exceeding cosmic scale). A still informative next-best strategy is to test a modification of the "wire-saving" adjacency rule for individual cells clustering into ganglia.

The adjacency rulc bccomcs an "introversion rule": "If neuron $a$ connects to $b$, then $a$ and $b$ are placed near each other, in particular, grouped together within the same ganglion, ceteris paribus." The alternative, null hypothesis here would be that neuron targets of connections from a given neuron are uniformly distributed or randomly scattered throughout the entire worm nervous system. One then seeks evidence that when $a$ connects to $b$, the probability is greater than chance that they in fact occupy the same ganglionic grouping. Table 5 was compiled using the same connection-counting conventions as Table 4; however, fiber tallies now are for numbers of connections between individual neurons, not ganglia. Table 5 shows that every one of the 10 ganglia is "introverted," with the proportion of connections from the ganglion into the ganglion itself always exceeding the ganglion's proportion of the total target neuron population (this trend in itself is significant, $p<0.01$ by a sign test). In addition, a $\chi^{2}$ test of the total population of connections, intraganglionic versus extraganglionic, shows that the "innerdirected" trend is highly significant, $p<0.0001$ : if a pair of connected neurons is arbitrarily selected, they are in fact sig- nificantly more likely than chance to turn out to occupy the same ganglion, in agreement with the above introversion clumping rule. When the introversion effect is similarly analyzed for each separate ganglion, it is statistically significant (approaching or exceeding $p<0.001$ ) for all but the two smallest ganglia, dorsal and dorsorectal (where, of course, connectivity data is most sparse); hence, the effect seems quite uniform among the ganglia. (Again, causal interpretation of how this phenomenon arises is a separate issue.)

It should be noted that, in several cases, intraganglionic connections are not made within the boundaries of a ganglion; for instance, all intraganglionic connections for the anterior, dorsal, and ventral ganglia are via the ring (see Table 4). However, such external intraganglionic connections still tend to be made in regions of adjacent or nearest neuropil; a ground rule of $C$. elegans fiber routing mechanisms seems to be that synapses tend to be formed en passant and within neuropil bundles (see White ct al., 1983).

Finally, there is evidence that component placement is so sensitive that even internal ganglion layout conforms to a CPO hypothesis - in particular, positioning of individual cell bodies within a ganglion that minimizes interganglionic connection lengths. For instance, one would expect, other things equal, a trend for somata making exclusively anterior extraganglionic connections to be located in the anterior half of a ganglion; correspondingly, neurons with external connections only to sites posterior to the ganglion would tend to be placed in the posterior half of the ganglion. And in fact, for pooled data from all ganglia, this internal structuring trend is highly significant $(p<0.001$ by a $\chi^{2}$ square test). The trend is particularly evident for all larger, elongated ganglia (such as ventral cord), as would be expected, since the greatest savings are possible there by adjusting anteroposterior sites of neurons. When this optimization of internal ganglion structure is taken into account (instead of treating all connections out of a ganglion as originating at the same centroid), it is evident that the difference in connection cost between the actual ganglion layout and alternate, runner- 
up layouts reported in the previous section will tend to increase appreciably.

\section{Discussion}

\section{Optimization mechanisms}

Given the observed phenomena of network optimization in the nervous system, how is this coordination of neural layout to minimize connection costs actually accomplished? Perhaps the simplest account would be in terms of "Nature, the Blind Watchmaker" just directly executing a brute-force trial and error check of all alternative layouts for the given connection matrix of the components. However, like many other combinatorial network optimization problems, CPO is much casicr to statc than to solve. Like Steiner tree, many versions of the problem have been proven to be "NP-hard" (Sahni and Bhatt, 1980; Frankle, 1987), that is, at least as difficult as any NP-complete problem (Lewis and Papadimitriou, 1978; Garey and Johnson, 1979). NP-hard, and NP-complete, problems are strongly conjectured to be intrinsically computationally complex; for each of the large number of problems of these types in a wide variety of domains, no efficient algorithm - that avoids in the worst case exhaustive search of all possible solutions - is known. To conveya sense of the computational intractability of exhaustive search for exact solution of CPO, it can be noted that the number of possible layouts of $n$ components on $n$ discrete positions (whether they form a one, two, or three-dimensional array) is $n$ ! For merely the layout problem of the 50 main areas of the human cerebral cortex, there are $50 !=3.04 \times 10^{64}$ alternative placement possibilities. The number of attoseconds $\left(10^{-18} \mathrm{sec}\right)$ in the 20 billion year history of the universe is $10^{35}$. Hence, if natural selection could test one layout per attosecond, all the time since dawn of the Universe, much less since emergence of life on Earth, would not suffice for this exhaustive search. Even parallelizing the process, with perfectly coordinated division of labor among every one of the estimated $10^{7}$ species now existing on Earth (May, 1988), still would not bring it near the realm of feasibility.

Exhaustive search is a simple, perfectly optimizing procedure for combinatorial problems, but even for cases of modest scale it is therefore not phylogenetically realizable. In current engineering practice, such computational paralysis is evaded by trading off perfectly correct and complete search algorithms for much less computationally costly "quick but dirty" probabilistic/approximation layout heuristics. Similarly, for natural selection, a multilevel design process is more plausible, where evolution "blindly" selects quick but dirty heuristics for in turn devising good brain layouts; later generations would inherit good design strategies of their forebears. (Various physical and biological constraints might conveniently happen to help cut down the total range of actually feasible layouts in the search.)

One possible candidate for such a network optimizing probabilistic/approximation heuristic process is of a "tug of war" type. For instance, there is evidence that neuron dendrite and axon arbor junctions are locally optimized in terms of Steiner tree volume-minimization, and that this is accomplished ontogenetically by just such a vector-mechanical energy minimization process (Cherniak, 1990a, 1992). Actual weights-andsprings analog hardware employing this type of process to solve practical single-component placement problems dates back to the turn of the century (Francis and White, 1974). For brain positioning to minimize total length of nerve connections, one could similarly picture each sensory and motor fiber behaving in effect over generations like a micro-spring stretching from sensor or muscle locations to the brain (cf. the "principle of neurobiotaxis" of Kappers et al., 1967). A corresponding layout procedure for the multiple-component-hence, combinatorial-problem in VLSI chip design is known as the "mesh of springs" heuristic, where each interconnection for a two-dimensional array of circuit elements can be represented as a spring. However, to avoid local-minima traps, such a system cannot simply be permitted to attain equilibrium in a single step; rather, the two axes of the array are each treated separately, and energy minimization proceeds by a relaxation method, in small stages (for review of this procedure in terms of a resistive nctwork analogy, sec Kuh and Ohtsuki, 1990; for discussion of "temperature-schedule" heuristics for chip layout, see Kirkpatrick et al., 1983).

Performance of multiple-element layout heuristics, even in well-controlled industrial settings, is still an open area of investigation -in particular, how good an approximation of the optimal solution can be expected how often. This point applies to evaluation of the "adjacency rule" described earlier, as well as to procedures of the energy-minimization type. The worm ganglion problem was small enough for brute-force search, and provides a benchmark that raises the possibility that neuroanatomical optimization heuristics may have to be quick, but perhaps cannot be very dirty. The evidence of microoptimization there suggests Nature's wiring heuristics might closely approximate best solutions.

Another area for future inquiry concerns implementation of concrete mechanisms for wiring optimization heuristics. Somc separation of explanatory levels is worth making along lines of the distinction in linguistics for theory of syntax between an abstract competence grammar and an actually employed, biologically real performance grammar (Chomsky, 1965). For instance, with regard to the adjacency rules and evidence supporting them, the question of direction of causation arises: does connection between two neural components in fact tend to lead to contiguous placement of them, or vice versa, or do both coevolve together, or are there different causal directions in different cases? A familiar observation in evolutionary theory is that biological designs tend to be "contraptions" not elegantly engineered de novo, but instead remodified from parts on hand (Gould, 1980). Thus, one could envisage a highly intricate process, at both phylogenetic and ontogenetic levels, of neural components just opportunistically connecting to near-neighbors, and then ad hoc adding on more remote connections as needed for functionality. One moral to draw from the observation of finely optimized coordination of component connections and positioning might then be a working hypothesis that there is greater latitude than many current models admit in how neural components can be interconnected and still function satisfactorily. Methodological interpretation of these results is discussed further in Cherniak (1994).

\section{References}

Achacoso T, Yamamoto W (1990) The AY neuroanatomic atlas of C. elegans for computation. Washington, DC: George Washington University Medical School.

Albertson D, Thomson J (1976) The pharynx of Caenorhabditis elegans. Philos Trans R Soc Lond [Biol] 275:299-325.

Bern M, Graham R (1989) The shortest-network problem. Sci Am 260:84-89.

Blinkov S, Glezer I (1968) The human brain in figures and tables: a quantitative handbook. New York: Plenum. 
Chalfie M, White J (1988) The nervous system. In: The nematode Caenorhabditis elegans (Wood W, ed), pp 337-391. Cold Spring Harbor, NY: Cold Spring Harbor Laboratory.

Cherniak C (1986) Minimal rationality. Cambridge, MA: MIT Press.

Cherniak C (1988) Undebuggability and cognitive science. Commun Assoc Comput Machinery 31:402-412.

Cherniak C (1990a) Local network optimization in the brain. University of Maryland Institute for Advanced Computer Studies Technical Report 90-90.

Cherniak C (1990b) The bounded brain: toward quantitative anatomy. J Cognit Neurosci 2:58-68.

Cherniak C (1991) Component placement optimization in the brain. University of Maryland Institute for Advanced Computer Studies Technical Report 91-98.

Cherniak C (1992) Local optimization of neuron arbors. Biol Cybern 66:503-510.

Cherniak C (1994) Philosophy and computational neuroanatomy. Philos Stud, in press.

Chomsky N (1965) Aspects of the theory of syntax. Cambridge, MA: MIT Press.

Francis R, White J (1974) Facility layout and location. Englewood Cliffs, NJ: Prentice-Hall.

Frankle J (1987) Circuit placement methods using multiple eigenvectors and linear probe techniques. $\mathrm{PhD}$ dissertation, University of California, Berkeley.

Garey M, Johnson D (1979) Computers and intractability: a guide to the theory of NP-completeness. San Francisco: Frecman.

Gould S (1980) The panda's thumb. New York: Norton.

Jouandet M, Tramo M, Herron D, Hermann A, Loftus W, Bazell J, Gazzaniga M (1989) Brainprints: computer-generated two-dimensional maps of the human cerebral cortex in vivo. J Cognit Neurosci $1: 88-117$.

Kappers C, Huber G, Crosby E (1967) Comparative anatomy of the nervous system of vertebrates. New York: Hafner.

Kirkpatrick S, Gelatt C, Vecchi M (1983) Optimization by simulated annealing. Science 220:671-680.

Krieg W (1963) Connections of the cerebral cortex. Evanston, IL: Brain Books.
Kuh E, Ohtsuki T (1990) Recent advances in VLSI layout. Proc IEEE 78:237-263.

Lewis H, Papadimitriou C (1978) The efficiency of algorithms. Sci Am 238:96-109.

May R (1988) How many species are there on Earth? Science 241: 1441-1449.

Price J (1987) The central olfactory and accessory olfactory systems. In: Neurobiology of taste and smell (Finger T, Silver W, eds), pp 179203. New York: Wiley.

Rakic P (1988) Specification of cerebral cortical areas. Science 241: 170-176.

Rosenquist A (1985) Connections of visual cortical areas in the cat. In: Cerebral cortcx, Vol 3 (Peters A, Jones E, eds), pp 81-117. New York: Plenum.

Sahni S, Bhatt A (1980) The complexity of design automation problems. In: Proceedings of the 17th Design Automation Conference, pp $402-411$.

Shepherd G (1979) The synaptic organization of the brain. New York: Oxford UP.

Soukup J (1981) Circuit layout. Proc IEEE 69:1281-1304.

Van Essen D (1985) Functional organization of primate visual cortex. In: Cerebral cortex, Vol 3 (Peters A, Jones, E, eds), pp 259-329. New York: Plenum.

Van Essen D, Maunsell J (1980) Two-dimensional maps of the cerebral cortex. J Comp Neurol 191:255-281.

White J, Southgate E, Thomson J, Brenner S (1976) The structure of the ventral cord of Caenorhabditis elegans. Philos Trans R Soc Lond [Biol] 275:327-348.

White J, Southgate E, Thomson J, Brenner S (1983) Factors that determine connectivity in the nervous system of Caenorhabditis elegans. Cold Spring Harbor Symp Quant Biol 48:633-640.

White J, Southgate E, Thomson J, Brenner S (1986) The structure of the nervous system of the nematode Caenorhabditis elegans. Philos Trans R Soc Lond [Biol] 314:1-340.

Wood W, ed (1988) The nematode Caenorhabditis elegans. Cold Spring Harbor, NY: Cold Spring Harbor Laboratory.

Young M (1992) Objective analysis of the topological organization of the primate cortical visual system. Nature 358:152-155. 